\title{
Emanometrische Messungen von Radon und Thoron in Bodenluft
}

\author{
H. Isratel - S. B.tornsson - S. Stiller $(*)$
}

Ricevuto il 12 Marzo 1962

\section{Finleitung.}

Die direkte messtechnische Trennung von Radon und Thoron in Luft ist bisher nur dann sicher möglich, wenn die zu untersuchende Luft ausser den beiden radioaktiven Edelgasen weder Induktionen noch (geladene und ungeladene) Suspensionen enthalt. Da dies nur selten - so z.B. bei Luftproben, die aus den Bodenkapillaren abgesaugt werden der Fall ist, ist die unmittelbare emanometrische Trennung von Radon und Thoron bisher nur bei Bodenluft in einfacher Weise moglich geworden.

Bei der Methode von J. Satterly $\left(^{(}\right)$wird die zu untersuchende Luftprobe zu einem definierten Zeitpunkt moglichst rasch in ein Ionisationsgefäss eingesaugt. Während der ersten Minuten setzt sich damn der Tonisationsstrom zusammen aus dem durch Radon bedingten langsam ansteigenden Strom (Kurve 1 in Abb. 1 oben) und dem mit der Halbwertszeit ron 54,5 sec abklingenden Anteil, der dur(h Thoron bedingt wird (Kurve 2 in $\mathrm{Abb} .1$ oben).

J. C. Sanderson ( $\left.{ }^{2}\right)$ saugt einen Bodenluftstrom bestimmter Geschwindigkeit durch einen Kondensator. Der Strom steigt ebenso wie im normalem Tonisationsgefäss in dem Masse an, wie sich die Induktionen RaA und RaC in ihm ablagern. Wird zu einem bestimmten Zeitpunkt der Luftstrom gestoppt, so klingt der im Apparat enthaltene ThoronAnteil mit 54,5 sec Halbwertszeit ab; der Strom sinkt also in der in $\Lambda$ bb. 1 unten skizzierten Weise und steigt bei erneutem Durchsaugen in gleicher Weise wieder an.

(*) Rheinisch-Westfalische Technische Hochschule - Aachen. 
In der Literatur finden sich ausser den Messungen von J. Satterly ( ${ }^{1}$ ) und J. C.. Sanderson ( ${ }^{2}$ ) bisher nur vereinzelt Angaben über den ThoronGehalt in Bodenluft. So berichten H. Israel und F. Becker $\left(^{3}\right)$ über Messungen in Bad Nauheim, die nach den genannten Methoden von Satterly und Sanderson in Tiefen von $0,5 \mathrm{~m}, 1 \mathrm{~m}$ und $2 \mathrm{~m}$ ausgeführt wurden. H. Norinder, A. Metnieks und R. Siksna $\left({ }^{4}\right)\left({ }^{5}\right)$ haben nach der Methode von Satterly in Jppsala Messungen in $90 \mathrm{~cm}$ Tiefe durchgefuhrt.
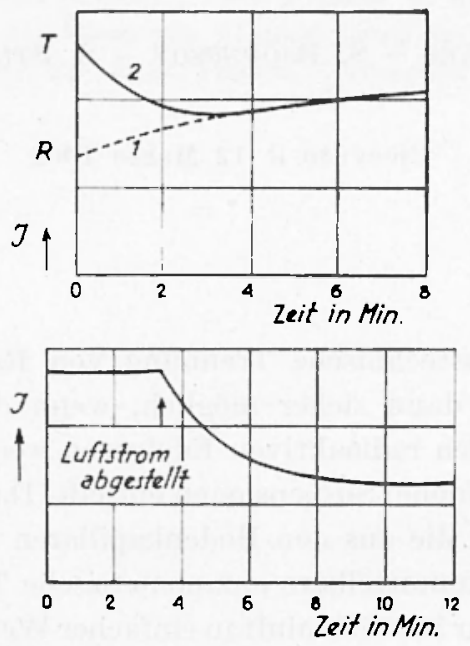

Abb. 1. - Schematische Darstellung der messtechnischen Trennung von Radon und Thoron nach $J$. Satterly (oben) und $J$. C. Sanderson (unten).

Im folgenden soll über eine Verfeinerung und messtechnische Frweiterung der Satterly-Methode und einige bisher damit erzielte Ergebnisse berichtet werden.

\section{TI. MLssmethonk.}

Die Darstellung der Satterly-Methode in Abb. 1 oben ist idealisiert und entspricht nicht ganz der Wirklichkeit. Denn es ist dabei angenommen, dass das Ionisationsgefäss zum Zeitpunkt t == 0 so rasch mit der zu untersuchenden Juft gefullt werden kann, dass in dieser Zeit kein Zerfall des Thorons stattfindet. Dies ist eine praktisch unertüllbare Forderung. Bei der kurzen Lebensdauer des Thorons wird wahrend der 
Füllzeit immer ein gewisser Verlust eintreten, dem Rechnung get ragen werden muss.

Ferner muss beim Einsaugen der Luftprobe in das vorher teilweise evakuierte Ionisationsgefäss die Abhängigkeit der Ionenbildung vom Druck Berücksichtigung tinden.

Beides zusammen bedingt einen Stromverlauf im Ionisationsgefäss, der von dem Schemabild der Abb. 1 oben erheblich abweicht.

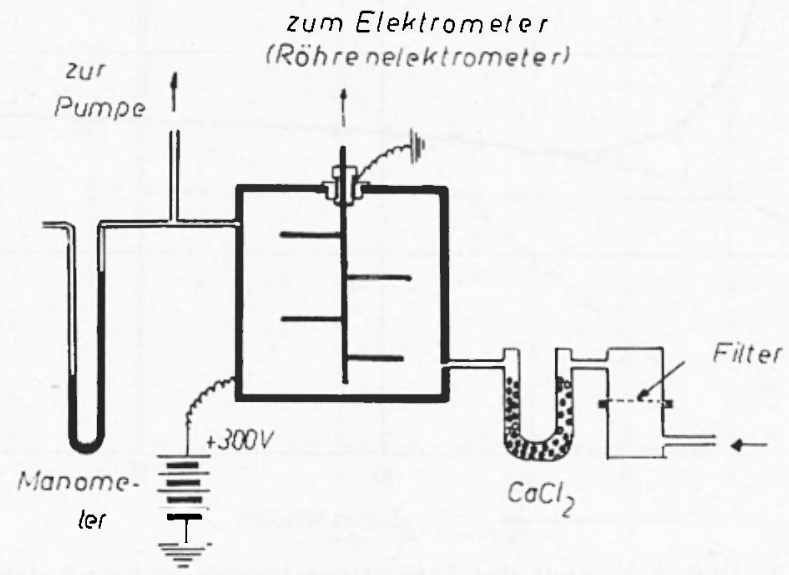

Abb. 2. - Messanordnung (schematisch)

Abb. 2 zeigt die Versuchsanordnung: Für die Messungen wurde ein Emanometer nach H. Israel $\left({ }^{6}\right)\left({ }^{*}\right)$ mit zwei Ionisationskammern von je etwa 201 Inhalt verwandt. Die Aufnahme der Bodenluft arfolgte nach der soe. "Sondenmethode " $\left.{ }^{7}\right)$ durch Ansaugen der Luft durch ein bis in die gewünschte Tiefe in den Boden eingeschlagenes dünnes Rohr. Zur Trocknung und Befreiung von Ionen, Staub und Induktionen wurde die Lult durch ein $\mathrm{CaCl}_{2}$-Rohr und ein Watte-, Glas- oder SchwebstofiFilter geleitet.

Abb. 3 zeigt eine mit dieser Anordnung am 9.5.1959 in Aachen gewonnene Messung. Dargestellt ist der zeitliche Verlauf des Ionisationsstromes wahrend der ersten 15 Minuten rom Beginn des Lufteinstromens an. Der Druckausgleich des vorher auf $400 \mathrm{~mm} \mathrm{Hg}$ evakuierten Gefässes erfolgte dabei mit etwa 20 sec Halbwertszeit. Das Gefass war also nach etwa 100 sec wieder zu $98,5 \%$ gefüllt. - Der Strom steigt

$\left(^{*}\right)$ Hersteller Firma spindler \& Hover, Göttingen. 
während der ersten $1 \frac{1}{2}$ Minuten rasch an. Dieses Ansteigen ist berlingt durch die Zunahme des Radons und Thorons in Gefass und durch die Zunahme des Ionisierungsvermögens der $\alpha$-Strahlung bei der Druckzunahme im Gefass.

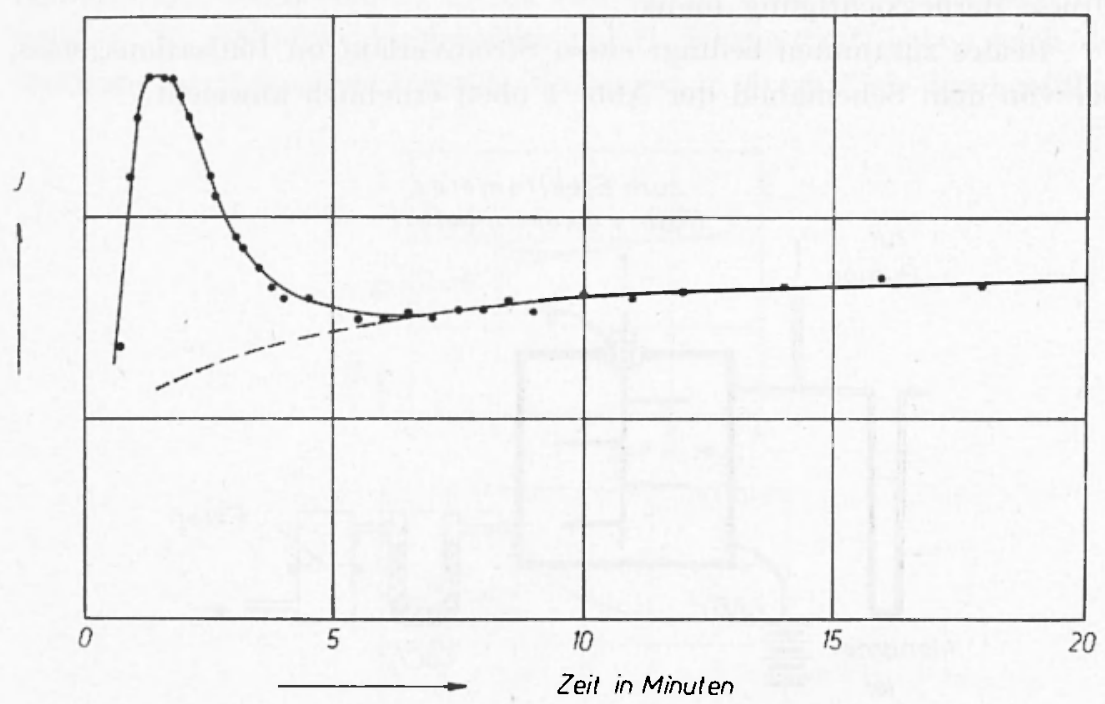

Abb. 3. - Zeitlicher Verlanf ıles Ionisationsstromes wälrend der ersten 20 Minuten (Messung am 9.5.1959).

Frst nach beendetem Einströmen zeigt dann der Stromverlauf das nach Abb. 1 oben zu erwartende Verhalten.

III. ANAIYSE.

Die analytische Zerlegung des gemessenen Stromverlaufes in den von Radon und den von Thoron herriihrenden Anteil erfolgt entweder in Näherung unter alleiniger Benutzung der nach der Gefässfullung gewonnenen Messwerte, Anwendung der Satterly-Analyse gemäss Abb. 1 oben auf diese und Zuruckextrapolation auf den Zeitpunkt $t=0$ (vergl. Abb. 1) oder exakter unter genauer rechnerischer Behandlung des Finstromungsvorganges und der dabei dem Gefass zugeführten ThoronMengen. Der Näherungswert ist, wie man sich leicht überzeugt. grosser als der wahre Wert und übertrifrt diesen umso meln, je langsamer das Finströmen erfolgt. 
Bei der Umrechnung der Stromwerte des Thoron-Anteiles in Curie ist zunächst zu beachten, dass das erste Umwandlungsprodukt ThA sehr kurzlebig ist $(0,16 \mathrm{sec}$ Halbwertszeit), wahrend der nächste a-Strahler (ThC) nur selur langsam über das langlebige ThB (10,6 h Halbwertszeit) entsteht. Man braucht deshalb zur Limrechmung nur die a-Ionisation von Thoron und ThA zu berücksichtigen.

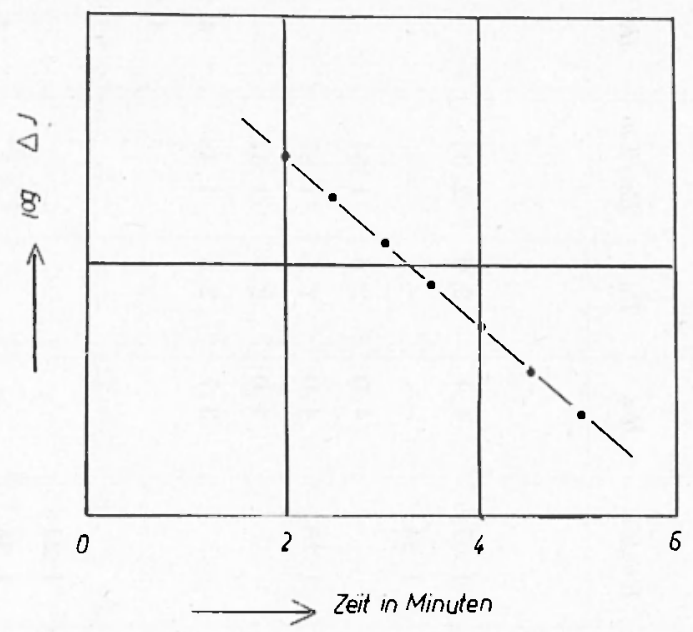

Abb. 4. - Thoron-Stromanteil bei der in Abb. 3 dargestellten Messung.

Der rasche Zerfall des ThA hat weiter zur Folge, dass den im lonisationsgefass entstehenden ThA-Atomen keine Zeit bleibt, sich ihrer positiven Ladung entsprechend (*) zur negativen Elektrode hin zu bewegen. Sie zerfallen vielmehr praktisch an der gleichen Stelle, wie ihre Mutteratome. Wir können also fur jeden Thoron-Zerfall $2 \alpha$-Strahlen annehmen.

Setzen wir nun für die $a$-Strahlung des Thorons den Wert von 6,4 MeV und fur. ThA den Wert von $6,9 \mathrm{MeV}$ an $\left(^{8}\right)$ und berechnen daraus unter der Anmahme, dass zur Bildung eines Ionenpaares $35 \mathrm{eV}$ benötigt werden, ihre Ionisationswirkung zu $1,83 \cdot 10^{5}$ für Thoron $\mathrm{bzw}$. $1,97 \cdot 10^{5}$ Tonempatre je $\alpha$-Strahl für ThA, so errechnet sich das Stromäquivalent $\mathrm{S}_{\mathrm{Tn}}$ für 1 Gurie Thoron (Tn) zu

$$
\begin{aligned}
S_{\mathrm{T} 11} & =3,7 \cdot 10^{10} \cdot(1,83+1,97) \cdot 10^{5} \cdot 4,8 \cdot 10^{-10} \\
& =6,75 \cdot 10^{6} \operatorname{Aes}(* * 6) \\
& =\left(2,25 \cdot 10^{-3} \mathrm{~A}\right)
\end{aligned}
$$

(*) Wir nelımen diese für das RaA bekannte Erfahrung aneh für 'T'h d an. (**) Elektrostatische Stromeinheiten. 
H. ISRAEL - S. BJÖRNSSON - S. STILLER

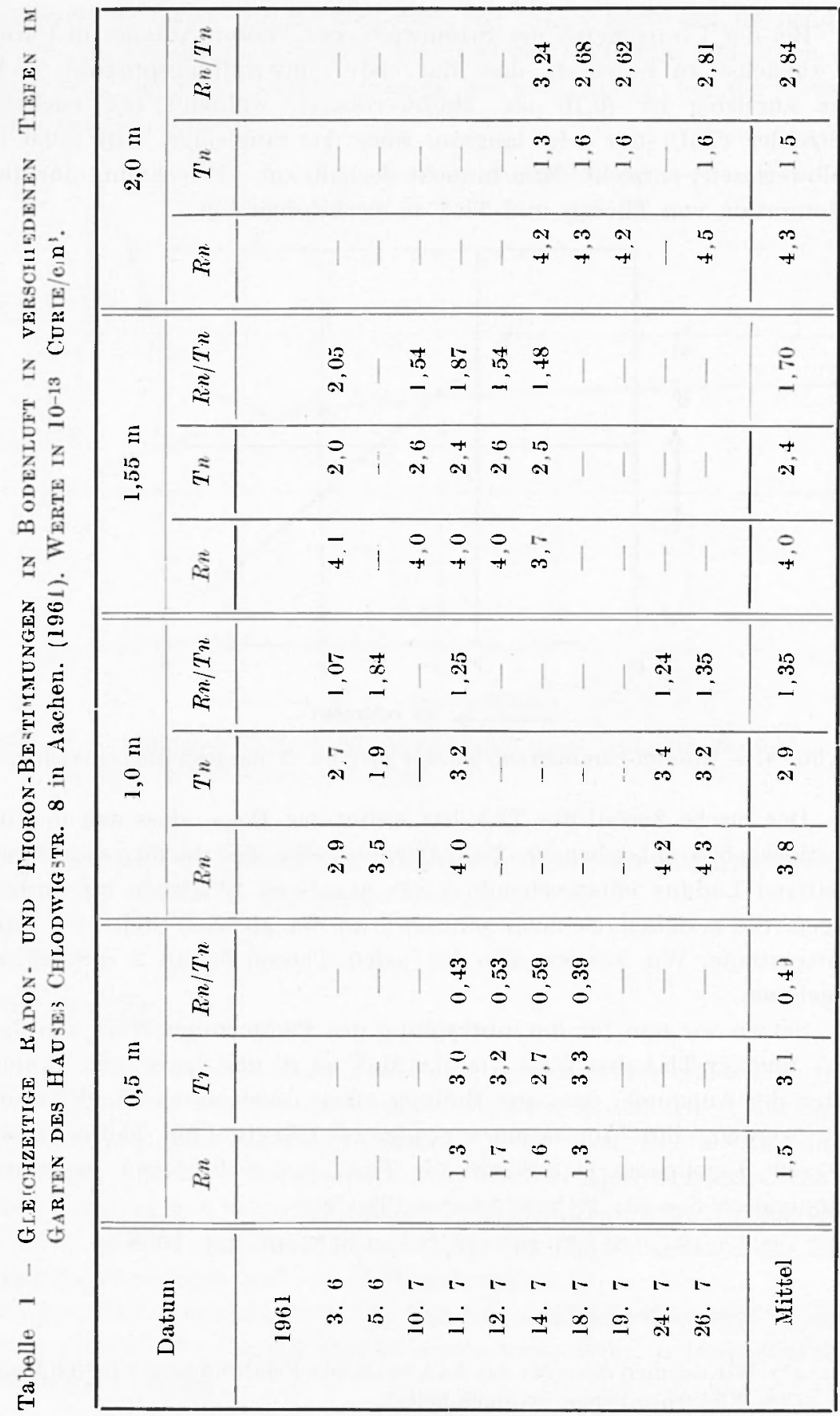


Tabelle 2 - Gleichzeitige Radon- und Thoron-Bestimingaen in Bodenluft in verschiedenen Tiefen im Garten des Meteorologiscien Observatoriums Aachen, Pipinstrasse 12. (1961). Werte in 10-13 Curie/cm³ (UNSICHERE WERTE IN KLAMMERN).

\begin{tabular}{|c|c|c|c|c|c|c|c|c|c|c|c|c|c|c|c|}
\hline \multirow{2}{*}{ Datum } & \multicolumn{3}{|c|}{$0,5 \mathrm{~m}$} & \multicolumn{3}{|c|}{$1,0 \mathrm{~m}$} & \multicolumn{3}{|c|}{$1,5 \mathrm{~m}$} & \multicolumn{3}{|c|}{$2,3 \mathrm{~m}$} & \multicolumn{3}{|c|}{$4,0 \mathrm{mI}$} \\
\hline & $R n$ & $T n$ & $R n / T n$ & $R n$ & $T n$ & $R n / T n$ & $R n$ & $T n$ & $R n / T n$ & $R \cdot n$ & $T n$ & $R n / T n$ & $R n$ & $T n$ & $R n / I^{\prime} n$ \\
\hline 1961 & & & & & & & & & & & & & & & \\
\hline 31.7 & 0,9 & 2,2 & 0,41 & 1,1 & $(0,7)$ & -- & 2,7 & 2,3 & 1,18 & 6,2 & 4,2 & 1,47 & 8,8 & 4,4 & 2,00 \\
\hline 1. 8 & 0,8 & 2,9 & 0,27 & 1,0 & 1,2 & 0,83 & 2,5 & 2,5 & 1,00 & 6,2 & 3,8 & 1,64 & 8,3 & 5,1 & 1,63 \\
\hline 2. 8 & 1,0 & 2,3 & 0,44 & - & 一 & -- & - & - & -- & - & - & - & - & - & - \\
\hline 3.8 & 1,0 & 2,5 & 0,40 & 1,1 & 1,2 & 0,84 & - & - & - & 6,0 & $(6,1)$ & - & - & - & - \\
\hline 4. 8 & 0,8 & 2,4 & 0,33 & 1,0 & 1,2 & 0,83 & 2,5 & 2.2 & 1,14 & 6,3 & 3,5 & 1,80 & 8,7 & 3,9 & 2,22 \\
\hline 7. 8 & 0,7 & 2,1 & 0,33 & 1,0 & 1,2 & 0,83 & 2,5 & 2,4 & 1,04 & 5,9 & 3,8 & 1,55 & 8,1 & 4,7 & 1,73 \\
\hline 8.8 & 0,6 & 2,3 & 0,26 & 1,0 & $(0,9)$ & - & - & - & - & 5,9 & 3,9 & 1,51 & 8,9 & 3,7 & 2,31 \\
\hline Mittel (*) & 0,8 & 2,4 & 0,35 & 1,0 & 1,2 & 0,83 & 2,6 & 2,4 & 1,09 & 6,1 & 3,9 & 1,60 & 8,6 & 4,4 & 1,98 \\
\hline
\end{tabular}

(*) Olne die eingeklemmerten Werte. 
IV. ERGIFIBNISSE.

Nach den beschriebenen Messverfahren wurlen in Aachen eine Reihe von Thoron-Bestimmungen in Bodenluft durehgeführt. Gemessen wurde in verschiedenen Bodentiefen in den Sandboden im Garten des Iauses Chlodwigstrasse 8 (Diensträume des Lehrgebietes Geophysik und

Tabelle 3 - Radon- und TIoron-Werte in Bodenluft in verschiedenex Tiefex is Bad Nauheim $\left.{ }^{3}\right)$. Werte in 10-13, Curie/cm.

\begin{tabular}{|c|c|c|c|c|c|c|c|c|c|}
\hline \multirow{2}{*}{ Datum } & \multicolumn{3}{|c|}{$0,5 \mathrm{~m}$} & \multicolumn{3}{|c|}{$1,0 \mathrm{mI}$} & \multicolumn{3}{|c|}{$2,0 \mathrm{~m}$} \\
\hline & $R n$ & $T_{n}$ & $R n / T n$ & $R n$ & $T n$ & $R n / T n$ & $R n$ & $T n$ & $R n / T n$ \\
\hline \multicolumn{10}{|l|}{1934} \\
\hline 16.10 & - & - & $\cdots$. & - & $\begin{array}{l}4,7 \\
6,6\end{array}$ & - & $\cdots$ & $\cdots$ & $\cdots$ \\
\hline 18.10 & $\ldots$ & $1,3\left(^{*}\right)$ & - & - & - & $\ldots$ & - & 4,5 & - \\
\hline 19.10 & $\cdots$ & $3,0\left(^{*}\right)$ & -- & - & - & $\ldots$ & - & $\cdots$ & - \\
\hline 25.10 & - & $\cdots$ & - & - & 10,6 & $\cdots$ & - & - & - \\
\hline 26.10 & - & - & - & - & - & - & - & 4,8 & $\ldots$ \\
\hline 30.10 & - & 4,7 & - & - & - & - & - & - & - \\
\hline Mittel & $28(* *)$ & 3,0 & 9,3 & $57^{* *}$ & 7,3 & 7.8 & $107^{* *}$ & 4,7 & 22,8 \\
\hline $\begin{array}{l}(*) \\
(* *)\end{array}$ & $\begin{array}{l}0,4 \mathrm{~m} \\
\text { Mitte }\end{array}$ & $\begin{array}{l}\text { Tiefe. } \\
\text { verte }\end{array}$ & ein & 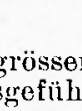 & 12 & 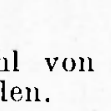 & Einz & & , \\
\hline
\end{tabular}

Meteorologie der Technischen Hochschule) und in der Nähe des Meteorologischen Observatoriums Aachen auf dem Wingertsberg im Staltpark Aachen. Die Auswertung erfolgte in der Regel nach dem Verfahren der genauen rechnerischen Berücksichtigung der Einstromungsverhältnisse. Weiter wurden die bei der Radon-Emanometrie üblichen Korrekturen für das verwandte Emanometer $\left({ }^{9}\right)$ auch auf die Thoron-NLessung mit diesem übertragen. Die Ergebnisse sind in den obigen Tabellen 1 und 2 zusammengestellt. Die 1. Kolonne enthalt jeweils den RadonGehalt, die 2. den Thoron-Gehalt, die 3. das Verhältnis Radon/Thoron 
fiir die betreflende Tiefe. Ian erkennt, dass sich die einzelnen Grössen an den beiden Messstellen verschieden verhalten. Der Radon-Gehalt steigt an beiden Orten rasch mit der Tiefe an. Der Thoron-Gehalt nimmi einmal (Tabelle 1.) mit der Tiefe ab, im anderen Fall (Tabelle 2) dagegen zu. Das Verhältnis zwischen Radon und Thoron steigt in beiden Fallen mit der Tiefe an, wenn auch in verschiedenem Masse.

In der weiteren Tabelle 3 sind zum Vergleich die in Bad Sauheim gewomnenen Werte $\left({ }^{3}\right)$ angegeben. Die Werte sind aus der seinerzeit verwandten Einheit in Curie umgerechnet. (*). Die Radon-und ThoronWerte sind dabei nicht gloichzeitig bestimmt, die ersten sind Mittelwerte aus einer grösseren Zahl von einzolnen Messungen an der gleichen Messstelle.

Das Material ist zwar noch viel zu klein, um eingehendere Schlussfolgerungen zuzulassen, immerhin aber kann man ihm einige llinweise entuehmen:

1. Nach theoretischer tberlegung $\left({ }^{9}\right)$ ist zu erwarten, dass in einem homogenen Boden, der in den hier in Frage kommenden Tiefen von einigen ILetern gleiche Zusammensetzung, gleiche Aktivität und gleiche Emanierungsfähigkeit besitzt, der Radon- und Thoron-Gehalt der Bodenluft exponentiell mit der Tiefe zunimmt. Fhre Konzentrationen nahern sich dabei nit einer Halbwertstiefe von 105, 5 cm fǜ Radlon und 1,35 cm für Thoron ihrem konstanten Tiefenwert. Der Thoron-Gelaalt, sollte also von etwa $10 \mathrm{~cm}$ Tiefe an nach unten konstant bleiben, das Verhältnis Radon/Thoron also ron dieser Tiefe an parallel zum Radon-Gehalt ansteigen.

Die Tabellen zeigen in dem raschen Anstieg des Radon-Gehaltes und der in ganzen zu erkennenden Zunahme des Radon/Thoron-Verhältnisses mit der Tiefe ein Verhalten, das qualitativ in der erwarteten Richtung liegt.

(*) Die seinerzeit als Thoron-Einheit gewählte "O. R.-Einheit " (die verallgemeinerte Definition des Curie existierte damals noch nicht) war definiert worden als die Thoronmenge, die mit $1 \mathrm{~g}$ Thorium im radioaktiven Gleichgewicht steht. Ihr Stromäquivalent ergab sich zu

$59,5 \times 10^{-2}$ Aes.

7usammen mit Gleichung 1 folgt daraus die Vmrechnung

I O. R.-Einheit entspricht

$\frac{59,5 \cdot 10^{-2}}{6,75 \cdot 10^{6}}=8,8 \cdot 10^{-8}$ Curie . 
2. Xach dem mittleren Wert des Uran- und Thorium-Gehaltes von Gesteinen und Böden $\left({ }^{10}\right)$ von etwa.

$$
3 \times 10^{-6}, U U \text { pro } g \text { Gestein }
$$

und

$$
10^{-5} g \text { Th pro } g \text { Gestein }
$$

folgt unter der Annahme, dass der Radon- und Thoron-Gehalt dem nach diesen Zahlen zu erwartenden Gleichgewichtswert entspricht, für mohrere Meter Tiefe einem Frwartungswert fïr das Radon/ThoronVerhaltnis von 0,93 .

Tatsächlich liegen aber die Werte an allen 3 Messorten durchweg höher. Fine Frklärung könnte vielleicht darin liegen, dass an diesen 3 Messstellen das Verhältnis von UrantThorium ein anderes ist, als es dem allgemeinen Frwartungswert entspricht. Auch kömnte möglicherweise das Emanielungsvermögen bei den beiden Emanationen Radon und Thoron ein verschiedenes sein.

3. Die Resultate von Bad Nauhejm sind trotz ihres geringen Unfanges dadurch interessant, dass bei wesentlich erhohten RadonGehalt der Thoron-Gehalt nicht entsprechend vergrössert ist. Man kamn aus dieser Tatsache den Hinweis entnehmen, dass offenbar der für die Bad Nauheimer Verhältnisse anzunehmende Vorgang (11) der RadonAnreichung in der Bodenluft, für die ein aus der "Quellenspalte" (Taunusmand-Verwerfung) nach oben dringender $\mathrm{CO}_{2}$ Strom als Träger verantwortlich gemacht wurde, sich im Thoron-Gehalt wegen des selur viel rascheren Zerfalls des Thoron nicht auswirken kann.

Die Arbeiten werden fortgesetzt.

\section{ZUSAMMENFASSUNG}

Mittels eines Emanometers nach H. Israel wurde im Sommer 1961 der Gehalt der Bodenluft an Rn und Tn an zwei Plätzen in verschiedener Tiefe nach der Satterly-Methode bestimmt. Die Ergebnisse werden mit ähnlichen Messungen von H. Israel und F. Becker in Bad Nauheim verglichen. - Die rasche Zunahme des Rn-Gehaltes und des Verhälnisses von Rn-Gehalt zu Tn-Gehalt mit der Tiefe entspricht der theoretischen Erwartung. Die Ergebnisse werden im einzelnen diskutiert. 


\section{RIASSUNTO}

Durante l'estate 1961 sono state misurate, in due localiti e a varie profondità, le concentrazioni di Rn $e$ Tn in terreno aereato. Le misure sono state eseguite con l'emanometro di Israel usando il metodo Satterly.

$I$ risultati sono paragonati a misure analoghe fatte da $H$. Israel $e$ $F$. Becker a Bad Nauheim. Il rapido aumento di $C_{R n}$ e del rapporto $C_{R n} / C_{T n}$ rispetto alla profondità in un terreno omogeneo, soddisfa qualitativamente all'aumento esponenziale ottenuto in teoria.

Vengono discussi $i$ risultati.

\section{$S D M M A R Y$}

The concentrations of $R n$ and $T n$ in soil-air were measured at two places and various depths during the summer 1961. The measurements were done with the emanometer of Israel using the Satterly-method.

The results are compared with similar measurements made by $H$. Israel and $F$. Becker in Bad Bauheim.

The rapid increase of $C_{R_{n}}$ and of the ratio $C_{R_{n}} / C_{T_{n}}$ does qualitatively agree with the theoretical exponential increase with depth in a homogeneous soil.

The results are discussed.

\section{IITERATUR}

(1) SATterLy J., The quantities of radium and thorium emanations contained in the air of certain soils "Proc. Cambridge Phil. Soc." 16, 514, 1910 1912.

(2) SANDERSON J. C., The probable influence of the soil on local atmospheric radioactivity. "Amer. J. Sci. " 32, 169-184. (1911). (Deutsche U'bersetzung s. "Pliys. Z." 13, 142 ff., (1912).

(3) Israel H. und Becker F., Die Bodenemanation in der Umgebung der Bad Nauheimer Quellenspalte. "Gerl. Beitr. Geophys." 44, 40-55, (1935).

(4) Norinder H., Metnieks A. und Siksna R., Radon content of the air in the soil at Uppsala. "Arkiv för Geofysik", 1, 571-579. (1952).

(5) Norinder H., Siksna R. und Metnieks A., Radon and thoron content of the soil-air at Almunge. "Geol. Foren. Stockholm Forh ", 74. II 4, 450-454, (1952). 
(6) IsRael H., Zur Methodih: klimatologischer Emanationsmessungen I: Öber ein neues Emanometer. "Balneologe", 1, 318-327. (1934).

(7) Isravi H., Radioaktive Messmethoden. In: "Taschenbuch der angewandten Geophysik" (Herausgegeben von R. Reich und R. v. Zwerger), 373-385, Leipzig, (1943).

( ${ }^{8}$ Daniel II., Die vier radioaktiven Zerfallsreihen. "Ergebn. d. exakt. Naturwiss ", 32, 188-197. (1959).

( $\left.{ }^{9}\right)$ Israki H., Die natürliche Radioaktivität in Boden Wasser und Luft. "Beitr. z. Phys. d. Atmosph." 30, 177-188. (1958).

(10) Landolt-Bornstein, Zahlenwerte und Funktionen, 6 Aufl, Bd. 3, 307 ff.

(11) Israer. II., Der Diffusionskoeffizient des Radons in der Bodenluft. (Bemerkung zu der Arbeit von E. Budde: "Bestimmung des Beweglichkeitskoffizienten der Radium-Emanation in Lockergesteinen ") "Ztschr. f. Geophys. ", 25, 104-108. (1959). - Der Diffusionskoeffizient des Radons in Bodenluft. (Abschliessende Stellungnahme zur Diskussion mit E. Budde). "Zeitschr. f. Geophys. ", 27, 13-17, (1961). 